\title{
2008-07
}

\section{A Framework for Uncertainty Quantification in Nonlinear Multi-body System Dynamics}

\author{
Makarand Datar, Dan Negrut \\ Simulation Based Engineering Laboratory \\ University of Wisconsin-Madison \\ D. Gorsich, D. Lamb \\ Tank-Automotive Research, Development and Engineering \\ Center (TARDEC) \\ Warren, $M I$
}

\section{September 2008}




\begin{abstract}
This technical report outlines a methodology for predicting the time evolution of complex nonlinear dynamic multi-body system in the presence of uncertain inputs. The focus is on ground vehicle systems, and the associated sources of uncertainty are multiple: road topology, friction coefficient at the road/tire interface, and aerodynamic force loading. Drawing on parametric maximum likelihood estimation, the methodology derived is general and can be applied to systematically study the impact of sources of uncertainty characterized herein by random processes. The proposed methodology is demonstrated through a study that characterizes the uncertainty induced in the loading of the lower control arm of an SUV type vehicle by uncertainty in the three dimensional road profile.
\end{abstract}




\section{Contents}

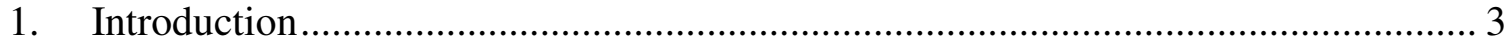

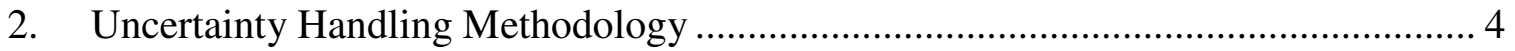

$2.1 \quad$ Parameter Estimation ............................................................................ 6

2.2 Covariance Function Selection ..................................................................... 7

3. Numerical Experiment: Parametric Model sensitivity...................................... 8

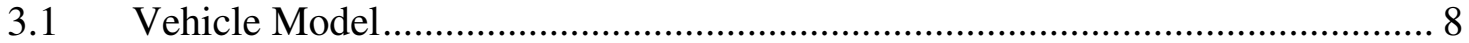

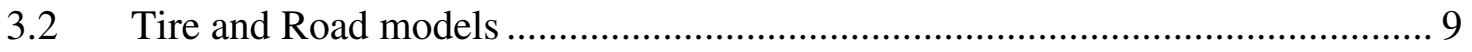

3.3 Numerical Results, Square Exponential ..................................................... 10

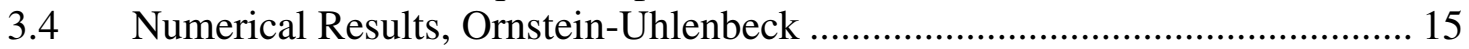

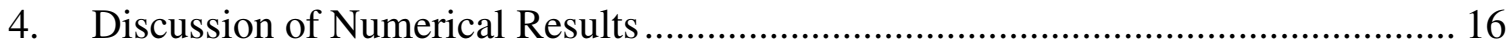

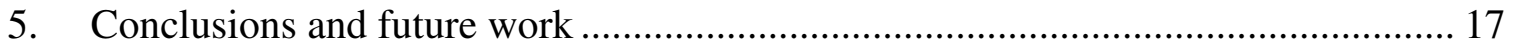

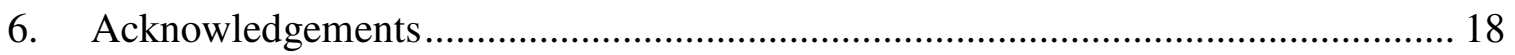

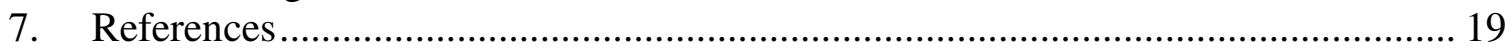




\section{Introduction}

The goal of this work is to establish an analytically sound and computationally efficient framework for quantifying uncertainty in the dynamics of complex multi-body systems. The motivating question for this effort is as follows: how can one predict an average behavior and produce a confidence interval vis-à-vis the time evolution of a complex multi-body system that is subject to uncertain inputs? Herein, of interest is answering this question for ground vehicle systems whose dynamics are obtained as the solution of a set of differential-algebraic equations (1996). The differential equations follow from Newton's second law. The algebraic equations represent nonlinear kinematic equations that constrain the evolution of the bodies that make up the system (Haug 1989).

The motivating question above is relevant for vehicle Condition-Based Maintenance (CBM) where the goal is to predict durability and the fatigue of system components. For instance, the statistics of lower control arm loading in a High-Mobility Multi-Wheeled Vehicle (HMMWV) obtained through a multi-body dynamics simulation become the input to a durability analysis that can predict in a stochastic framework the condition of the part and recommend or postpone system maintenance. A stochastic characterization of system dynamics is also of interest in understanding limit behavior of the system. For instance, providing in real time a confidence interval for certain maneuvers are useful in assessing the control of a vehicle operating on icy road conditions.

Vehicle dynamics analysis under uncertain environment conditions, e.g. road profile (elevation, roughness, friction coefficient) and aerodynamic loading, requires approaches that draw on random functions. The methodology is substantially more involved than that which is required for handling uncertainty that enters the problem through discrete design parameters associated with the model. For instance, uncertainty in suspension spring stiffness or damping rates can be handled through random variables. In this case, methods such as the polynomial chaos (PC), see, for instance, (Xiu; Karniadakis 2002) are suitable provided the number of random variables is small. This is not the case here, since a discretization of the road leads to a very large number of random variables (the road attributes at each road grid point). Moreover, the PC methodology requires direct access and modification of the computer program used to run the deterministic simulations to produce first and second order moment information. This represents a serious limitation if relying on commercial off-the-shelf (COTS) software, which is most often the case in industry when running complex high-fidelity vehicle dynamics simulation.

In conjunction with Monte Carlo analysis, the alternative considered herein relies on random functions to capture uncertainty in system parameters and/or input. Limiting the discussion to three-dimensional road profiles, the methodology samples a posterior distribution that is conditioned on available road profile measurements. Two paths can be followed to implement this methodology; the first draws on a parametric representation of the uncertainty, the second being nonparametric in nature. The latter approach is general yet expensive to implement. It can rely on smoothing techniques (nonparametric regression) that use kernel estimators such as Nadaraya-Watson or variants, see, for instance, (Wasserman 2006). The parametric approach is used in this paper by considering Gaussian Random Functions as priors for the road profiles. Furthermore, the discussion will be limited to stationary processes although current research is also investigating the nonstationary case. 
As always the case, the use of a parametric model raises two legitimate questions: why a particular parametric model, and why is it fit to capture the statistics of the problem. Gaussian Random Functions (GRF) are completely defined by their correlation function, also known as variogram (Adler 1990; Cramér; Leadbetter 1967). Consequently, scrutinizing the choice of a parametric GRF model translates into scrutinizing the choice of correlation function. There are several families of correlation functions, the more common being exponential, Matérn, linear, spherical, and cubic (see, for instance (Santner et al. 2003)). In this context and in order to demonstrate the proposed framework for uncertainty quantification in multi-body dynamics, a representative problem will be investigated in conjunction with the selection of a GRF-based prior. Specifically, an analysis will be carried out to assess the sensitivity of the response of a vehicle to uncertainty in system input, here a road profile. The outcome of interest will be the load history for the lower-control arm of an HMMWV, a key quantity in the CBM of the vehicle. The parametric priors considered are (i) a GRF with a squared exponential correlation function, and (ii) the Ornstein-Uhlenbeck process. Pronounced sensitivity of the statistics of the loads acting on the lower control arm with respect to the choice of parametric model would suggest that serious consideration needs to be given to the nonparametric route, where the empirical step of variogram selection is avoided at the price of a more complex method and increase in simulation time.

\section{Uncertainty Handling Methodology}

The discussion herein concerns handling uncertainty in spatial data. This situation commonly arises when limited information is used to generate road profiles subsequently

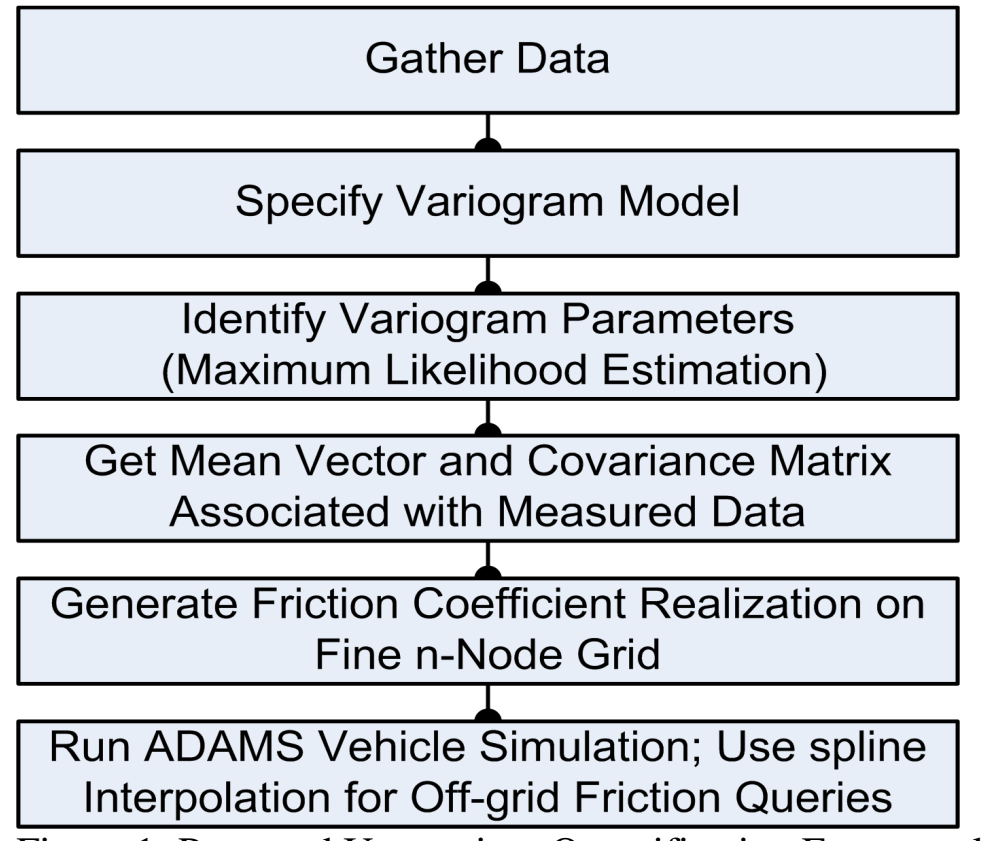

Figure 1: Proposed Uncertainty Quantification Framework used in the dynamic analysis of a ground vehicle. Similarly addressed, the handling uncertainty in aerodynamic loads is not of primary interest in this study and will be omitted.

The uncertainty quantification framework proposed is described in Figure 1. An assumption is made that learning data has been made available as the result of field measurements. Referring to Figure 2, the measured data is available on a "coarse" measurement grid. For dynamic analysis, road information is ideally available everywhere on the road as a continuous data. As this is not possible, data is 
provided on a fine grid (right image in Figure 2). If working with a parametric model, a correlation function is selected and a learning stage follows. Its outcome, a set of hyperparameters associated with the correlation function, is instrumental in generating the mean and covariance matrix ready to be used to generate sample road surfaces on the user specified fine grid.

The learning data that we have is the data about road elevation as a discrete function of a road's $\mathrm{x}$-y co-ordinates, which is a two-dimensional problem $(d=2)$. An example of such a road can be seen in Figure 2.
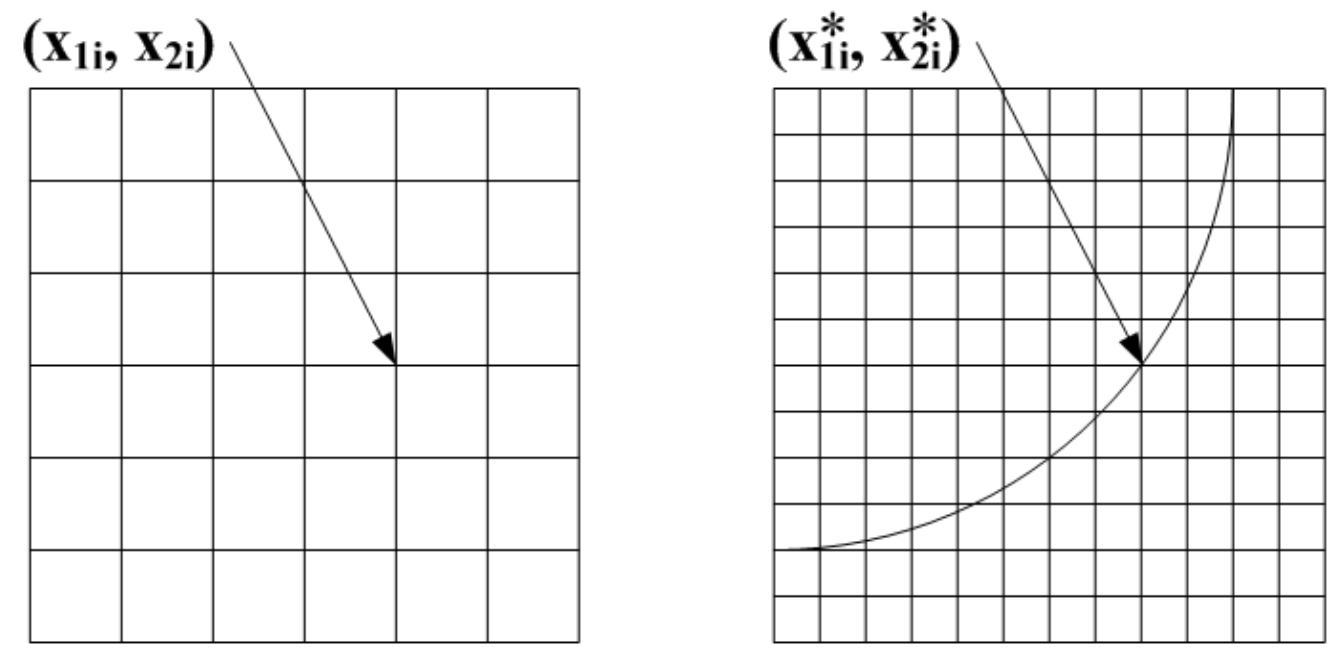

Figure 2. Coarse grid for learning, and fine grid employed in sampling for Monte Carlo analysis. Here $d=2$.

The use of Gaussian Random Functions (GRF) or processes are a very versatile approach for the simulation of infinite dimensional uncertainty. In general, a spatially distributed random variable $\mathbf{y}(\mathbf{x}), \mathbf{x} \in \mathbb{R}^{d}$, is a GRF with mean function $m\left(\mathbf{x} ; \theta_{1}\right)$ and correlation function $k\left(\mathbf{x}, \mathbf{x}^{\prime} ; \theta_{2}\right)$ if, for any set of space points $\mathbf{X}=\left\{\mathbf{x}_{1}, \mathbf{x}_{2}, \ldots, \mathbf{x}_{M}\right\}$,

$$
\mathbf{y}(\mathbf{X})=\left(\begin{array}{c}
y_{1} \\
y_{2} \\
\vdots \\
y_{M}
\end{array}\right) \sim N\left(\mathbf{m}\left(\mathbf{X} ; \theta_{1}\right), \mathbf{K}\left(\mathbf{X}, \mathbf{X} ; \theta_{2}\right)\right)
$$

Here $\mathbf{m} \in \mathbb{R}^{M}, \mathbf{K} \in \mathbb{R}^{M \times M}$, and $N(\mathbf{m}, \mathbf{K})$ is the M-variate normal distribution with mean $\mathbf{m}$ and covariance $\mathbf{K}$ given by

$$
\mathbf{m}\left(X ; \theta_{1}\right)=\left(\begin{array}{c}
m\left(x_{1}, \theta_{1}\right) \\
m\left(x_{2}, \theta_{1}\right) \\
\vdots \\
m\left(x_{M}, \theta_{1}\right)
\end{array}\right)
$$


$\mathbf{K}\left(\mathbf{X}, \mathbf{X}^{\prime} ; \theta_{2}\right)=\left(\begin{array}{ccc}k\left(x_{1}, x_{1}^{\prime} ; \theta_{2}\right) & \cdots & k\left(x_{1}, x_{N}^{\prime} ; \theta_{2}\right) \\ k\left(x_{2}, x_{1}^{\prime} ; \theta_{2}\right) & \cdots & k\left(x_{2}, x_{N}^{\prime} ; \theta_{2}\right) \\ \vdots & \vdots & \vdots \\ k\left(x_{M}, x_{1}^{\prime} ; \theta_{2}\right) & \cdots & k\left(x_{M}, x_{N}^{\prime} ; \theta_{2}\right)\end{array}\right)$,

where $\mathbf{X}^{\prime}=\left\{\mathbf{x}_{1}^{\prime}, \mathbf{x}_{2}^{\prime}, \ldots, \mathbf{x}_{N}^{\prime}\right\}$. The hyper-parameters $\theta_{1}$ and $\theta_{2}$ associated with the mean and covariance functions are obtained from a data set $\mathbf{y}(D)$ at nodes $D=\left\{d_{1}, \ldots, d_{M}\right\}$. The posterior distribution of the variable $\mathbf{y}(S)$ at node points $S=\left\{s_{1}, \ldots, s_{N}\right\}$, consistent with $\mathbf{y}(D)$, is $N\left(\mathbf{f}^{*}, \mathbf{K}^{*}\right)$ (Rasmussen; Williams 2006), where

$\mathbf{f}^{*}=\mathbf{K}\left(S, D ; \theta_{2}\right) \mathbf{K}^{-1}\left(D, D ; \theta_{2}\right)\left(\mathbf{y}(D)-\mathbf{m}\left(D ; \theta_{1}\right)\right)+\mathbf{m}\left(S ; \theta_{1}\right)$

$\mathbf{K}^{*}=\mathbf{K}\left(S, S ; \theta_{2}\right)-\mathbf{K}\left(S, D ; \theta_{2}\right) \mathbf{K}^{-1}\left(D, D ; \theta_{2}\right) \mathbf{K}\left(D, S ; \theta_{2}\right)$

The key issues in sampling from this posterior are a) how to obtain the hyper-parameters from data, and b) how to sample from $N\left(\mathbf{f}^{*}, \mathbf{K}^{*}\right)$ especially in the case where $M$ is very large. The classical way to sample relies on a Cholesky factorization of $\mathbf{K}^{*}$, a costly order $O\left(M^{3}\right)$ operation. The efficient sampling question is discussed in (Anitescu et al. 2008). A brief description of the hyper-parameter calculation follows.

\section{$2.1 \quad$ Parameter Estimation}

The method used herein for the estimation of the hyper-parameters from data is maximum likelihood estimation (MLE) (Rasmussen; Williams 2006). The method relies on the maximization of the log-likelihood function. In the multivariate Gaussian with mean $\mathbf{m}(\theta) \in \mathbb{R}^{M}$ and covariance matrix $\mathbf{K}(\theta) \in \mathbb{R}^{M \times M}$ case, the log-likelihood function assumes the form

$\log p(\mathbf{y} \mid \theta)=-\frac{1}{2} \mathbf{W}^{T} \mathbf{K}(\theta)^{-1} \mathbf{W}-\frac{1}{2} \log |\mathbf{K}(\theta)|-\frac{M}{2} \log 2 \pi$

Here $\mathbf{W}=\mathbf{y}-\mathbf{m}(\theta)$ and $\mathbf{y}$ is the observed data. Note that the dependence on the hyperparameters $\theta$ appears by means of the coordinates $\mathbf{x}$, and $\theta=\left\{\theta_{1}, \theta_{2}\right\}$. The gradients of the likelihood function can be computed analytically (Rasmussen; Williams 2006):

$$
\begin{aligned}
& \frac{\partial}{\partial \theta_{1 j}} \log p(\mathbf{y} \mid \theta)=-\left(\frac{\partial}{\partial \theta_{1 j}} \mathbf{m}(\theta)\right)^{T} \mathbf{K}(\theta)^{-1} \mathbf{W} \\
& \frac{\partial \log p(\mathbf{y} \mid \theta)}{\partial \theta_{2 j}}=\frac{1}{2} \operatorname{tr}\left(\left(\mathbf{K}(\theta)^{-1} \mathbf{W}\left(\mathbf{K}(\theta)^{-1} \mathbf{W}\right)^{T}-\mathbf{K}(\theta)^{-1}\right) \frac{\partial \mathbf{K}(\theta)}{\partial \theta_{2 j}}\right)
\end{aligned}
$$

MATLAB's fsolve function, which implements a quasi-Newton approach for nonlinear equations, was used to solve the first order optimality conditions $\frac{\partial \log p(\mathbf{y} \mid \theta)}{\partial \theta_{1 j}}=0$ and $\frac{\partial \log p(\mathbf{y} \mid \theta)}{\partial \theta_{2 j}}=0$ to determine the hyper-parameters $\theta_{1}$ and $\theta_{2}$. The entire approach hinges 
at this point upon the selection of the parametric mean and covariance. It is common to select a zero mean prior $\mathbf{m} \equiv 0$, in which case only the $\theta_{2 j}$ hyper-parameters associated with the covariance matrix remain to be inferred through MLE.

\section{2}

\section{Covariance Function Selection}

The parametric covariance function adopted determines the expression of the matrix $\mathbf{K}(\theta)$ of the previous subsection and it requires an understanding of the underlying statistics associated with the data. In what follows, the discussion focuses on four common choices of correlation function: squared exponential (SE), Ornstein-Uhlenbeck (OU) (Uhlenbeck; Ornstein 1930), Matérn (Matérn 1960), and neural network (NN) (Neal 1996).

The SE correlation function assumes the form

$$
k\left(x, x^{\prime} ; \theta_{2}\right)=\exp \left(-\left[\frac{\left(x_{1}-x_{1^{\prime}}\right)}{\theta_{21}}\right]^{2 / \gamma}-\left[\frac{\left(x_{2}-x_{2^{\prime}}\right)}{\theta_{22}}\right]^{2 / \gamma}\right),
$$

where $\gamma=1$. The hyper-parameters $\theta_{21}$ and $\theta_{22}$ are called the characteristic lengths associated with the stochastic process and they control the degree of spatial correlation; large values of these coefficients lead to large correlation lengths, while small values reduce the spatial correlation leading in the limit to white noise, that is, completely uncorrelated data. The SE is the only continuously differentiable member of the family of exponential GRF. As such, it is not commonly used for capturing road profiles, which are typically not characterized by this level of smoothness. To this end, Stein (Stein 1999) recommends the Matérn family with the correlation function

$$
k\left(r ; \theta_{2}\right)=\frac{2^{1-v}}{\Gamma(v)}\left(\frac{\sqrt{2 v} r}{l}\right)^{v} K_{v}\left(\frac{\sqrt{2 v} r}{l}\right),
$$

with positive parameters $v$ and $l$, where $K_{v}$ is the modified Bessel function. The degree of smoothness of the ensuing GRF can be controlled through the parameter $v$ : the corresponding GRF is $p$-times differentiable iff $v>p$. Note that selecting $\gamma=2$ in Eq. (4) leads to the OU random process, which is a nonsmooth process although not as versatile as the Matérn family.

The three covariance models discussed so far: SE, OU, and Matérn are stationary. Referring to Eq. (1), this means that for any set of points $\mathbf{X}=\left\{\mathbf{x}_{1}, \mathbf{x}_{2}, \ldots, \mathbf{x}_{M}\right\}$, where $M$ is arbitrary, and for any vector $\mathbf{h} \in \mathbb{R}^{\mathbf{d}}, \mathbf{y}(\mathbf{X})$ and $\mathbf{y}(\mathbf{X}+\mathbf{h})$ always have the same mean and covariance matrix. In particular, when $M=1$, this means that the GRF should have the same mean and variance everywhere. Clearly, the stationary assumption does not hold in many cases. For vehicle simulation, consider the case of a road with a pothole in it, which cannot be captured by stationary processes. A versatile nonstationary neural network covariance function has been proposed by Neal (1996): 
$k\left(\mathbf{x}, \mathbf{x}^{\prime} ; \theta_{2}\right)=\frac{2}{\pi} \sin ^{-1}\left(\frac{2 \tilde{\mathbf{x}}^{T} \Sigma \tilde{\mathbf{x}}^{\prime}}{\sqrt{\left(1+2 \tilde{\mathbf{x}}^{T} \Sigma \tilde{\mathbf{x}}\right)\left(1+2 \tilde{\mathbf{x}}^{\prime T} \Sigma \tilde{\mathbf{x}}^{\prime}\right)}}\right)$,

where $\tilde{\mathbf{x}}=\left(1, x_{1}, \ldots, x_{d}\right)^{T}$ is an augmented input vector; the symmetric positive definite matrix $\Sigma$ contains the parameters associated with this GRF and are determined based on the MLE approach described in 2.1. In this context, Rasmussen and Williams (2006) suggest $\Sigma=\operatorname{diag}\left(\sigma_{0}, \sigma_{1}, \ldots, \sigma_{d}\right)$. Note that for the road profile problem $d=2$.

Using this parameter estimation approach, a mean and co-variance function for Gaussian processes is determined. This is then used to generate new roads which are statistically equivalent to the road used in the learning process. Figure 5. shows the vehicle model in ADAMS/Car on two such roads.

\section{Numerical Experiment: Parametric Model sensitivity}

The numerical experiments carried out illustrate how the proposed uncertainty

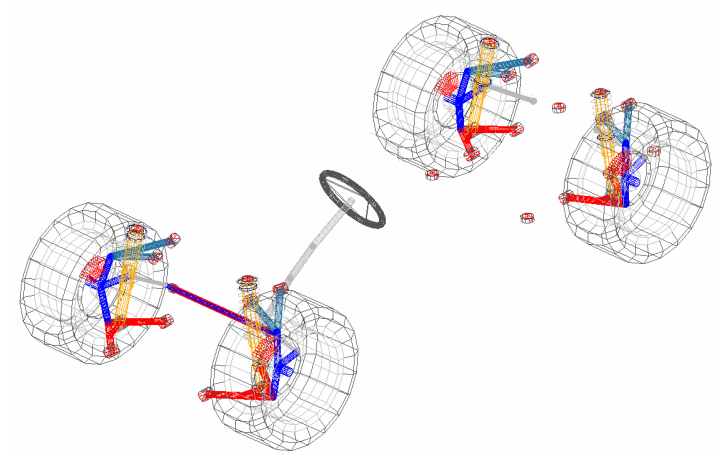
quantification framework is used to predict an average behavior and produce a confidence interval vis-à-vis the time evolution of a complex multi-body system. A high-fidelity nonlinear ground vehicle model is considered, and its time evolution is subject to data uncertainty stemming from measurements of the road profile. This setup was chosen due to its relevance in CBM, where the interest is the statistics of the loads acting on the

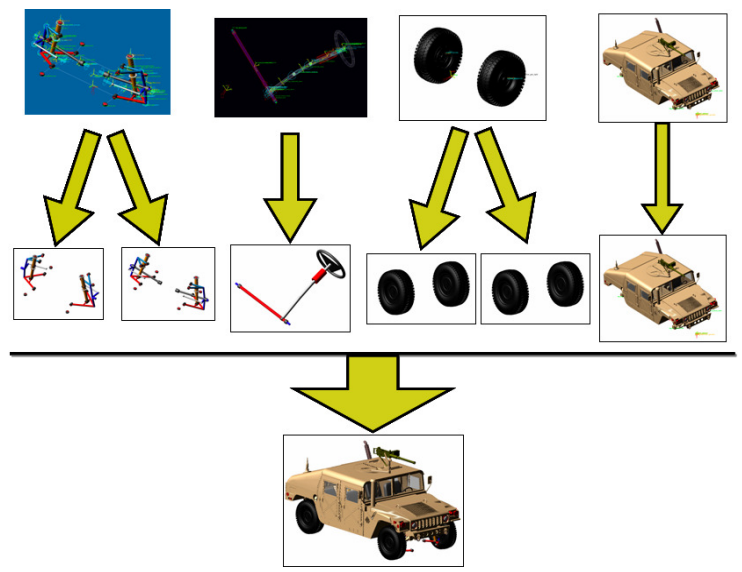

Figure 3. Up: Vehicle model, (no chassis geometry shown). Down: Schematic of how different subsystems are assembled to make a full vehicle model. vehicle for durability analysis purposes. Note that a similar analysis is carried out for a simplified scenario that does not involve the MLE learning stage by Schmitt et al. (2008) in conjunction with quantifying the uncertainty of vehicle dynamics when running on icy roads with a stochastic distribution of the tire/road friction coefficient.

\section{$3.1 \quad$ Vehicle Model}

The vehicle of interest in this work is a SUV-type vehicle similar, although not identical, to the Army's High Mobility Multi-Wheeled Vehicle (HMMWV). A high-fidelity model of the vehicle was 
generated in ADAMS, a widely used COTS that contains a template library, ADAMS/Car, dedicated to ground vehicle modeling and simulation. The steering system is of the rack-and-pinion type. The vehicle is equipped with an Ackerman type suspension system. The front and rear suspensions have the same topology but different link lengths. The location of the suspension subsystem is parameterized with respect to the chassis of the vehicle to allow for an easy editing of the assembly topology. Although not a topic of interest in this paper that deals with infinite dimensional stochastic processes such as uncertainty in road profile, this parameterization of the suspension allows for a Monte-Carlo based approach to quantify the uncertainty in vehicle dynamics produced by this model subcomponent.

Figure 3 shows the topology of a vehicle with front and rear suspension, wheels, and steering subsystems. Different vehicle subsystems are individually modeled and are integrated together to form a full vehicle model. The chassis of the vehicle is modeled as a single component having appropriate mass-inertia properties. ADAMS/Flex is typically used to add compliance to the steering and/or chassis components of the vehicle. This process makes use of modal neutral format (MNF) files created using a third party finite element package such as ABAQUS.

\subsection{Tire and Road models}

Two types of external forces act under normal conditions on the vehicle and influence its dynamics: forces at the tire-road interface and aerodynamic forces. Given the range of speeds at which the considered ground vehicle is driven, the former forces are prevalent and high-fidelity simulation depends critically on their accurate characterization. In this context, the SUV configuration of interest is designed to drive over a variety of terrains, from flat smooth pavement to off-road conditions.

The FTire tire model (Gipser 2005) serves as a sophisticated tire force element. It can be used in multi-body system models for vehicle handling and comfort investigations as well as for durability studies on even or uneven roadways. Specifically, FTire is designed for vehicle comfort simulations on surfaces that posses extremely short wavelengths. At the same time, it serves as a physics-based, highly nonlinear, dynamic tire model for investigating handling characteristics under the above-mentioned excitation conditions. FTire is fast (typically only 5 to 20 times slower than real-time) and numerically robust. The tire belt is described as an extensible and flexible ring carrying bending loads, elastically founded on the rim by distributed, partially dynamic stiffness values in radial, tangential, and lateral directions. The tire model is accurate up to relatively high frequencies (up to $120 \mathrm{~Hz}$ ) both in longitudinal and lateral directions. It works out of, and up to, a complete standstill, without any additional computing effort or model switching. It is also applicable in demanding applications such as ABS braking on uneven roadways.

In terms of the road models supported, they should accommodate the particular tire model they work in conjunction with. For Magic Formula tire models this is not an issue, since the road description is very basic. In this and the case of the Fiala tire model, the 
road environment of choice is the one provided by ADAMS, where the road is defined by a text based data file (rdf). This rdf file contains the information about road size, type (flat, periodic obstacles, stochastic 3D) and coefficients of friction over the road surface. Defining a flat road is trivial and an obstacle in the road (curb, roof-shaped) can be defined by specifying the size and shape of the obstacle in the rdf file. For roads with varying elevations in both lateral and longitudinal directions, a tessellated road definition is supported in ADAMS. A tessellated road is described by a set of vertices/nodes, which are grouped in sets of three to create a triangulated mesh that describes the entire road surface. A coefficient of friction can be specified for each triangle. This road definition works well with both synthesized and measured road data. The disadvantage of this road definition is that for each time step, the simulation has to check every triangle for contact with the tire patch leading to a major computational bottleneck (extremely long simulation times for large road profiles with high resolution). In order to simulate large road profiles, the regular grid road (rgr) file format is used in conjunction with FTire. A conversion from tessellated to the new rgr format leads to smaller file sizes and significantly reduces CPU time per simulation step in that the CPU time required for the tire/terrain interaction is independent of the dimensions (length/width) of the road profile.

\subsection{Numerical Results, Square Exponential}

The SUV model discussed in subsection 3.1 was equipped with a set of four tires generated in the FTire modeling and simulation package discussed in subsection 3.2. This complete vehicle model was exercised through a straight-line maneuver over a road

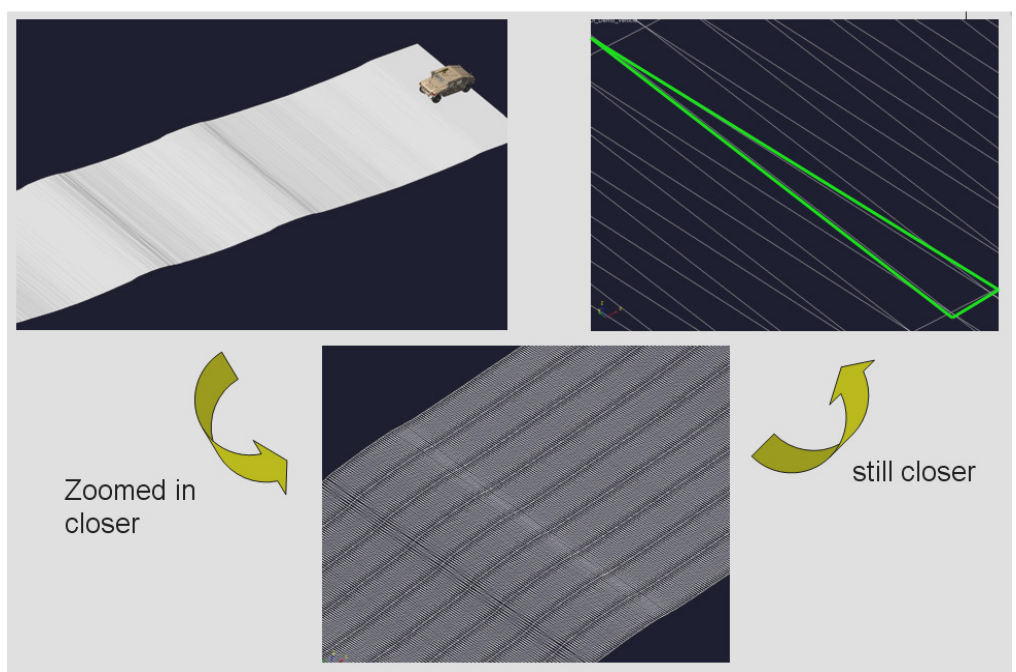

Figure 4: UP: Road tessellation, Right: Triangulation applied to a road profile

vehicle configuration. profile for which information is available on a grid as follows (see also Figure 4): in the $\mathrm{x}-$ direction, information is provided every 0.25 feet in 180 slices. In the $y-$ direction, the data is provided at a distance of four feet apart in 3 slices. The length of the course in the $\mathrm{x}$-direction was approximately 45 feet. The width of the road was 8 feet. Although not reported here, simulations up to one mile long have been run using this 
The stochastic analysis proceeded according to the work flow in Figure 1. A road

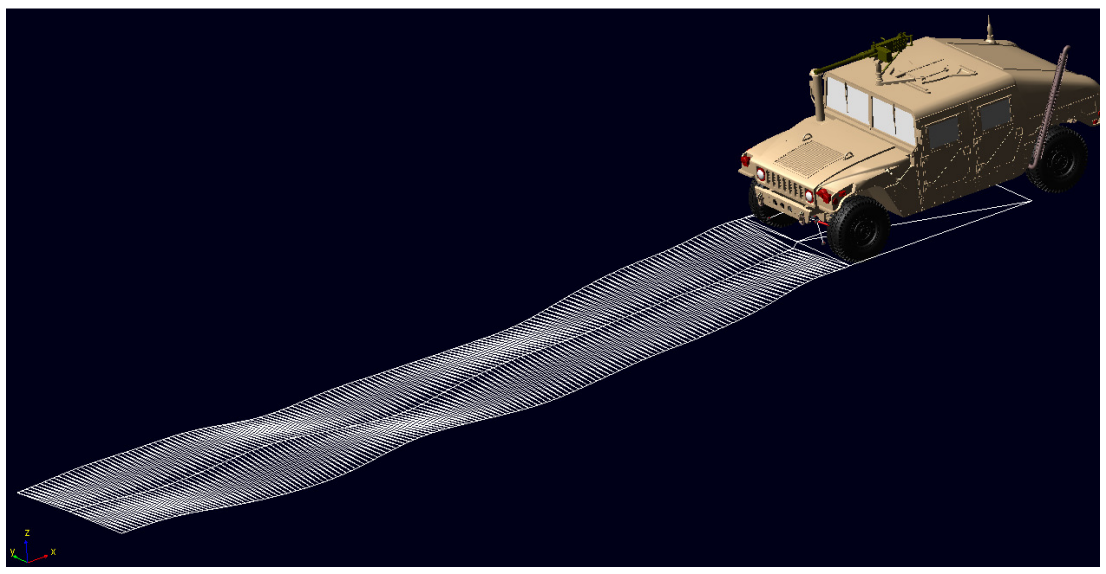
profile was provided and considered the outcome of a set of field measurements on a $180 \mathrm{X} 3$ grid as indicated above. MLE was carried out, the resulting characteristic lengths were $\theta_{x}=4.5355$ and $\theta_{y}=0.8740, \quad$ which are identified in Eq. (4) with $\theta_{21}$ and $\theta_{22}$,

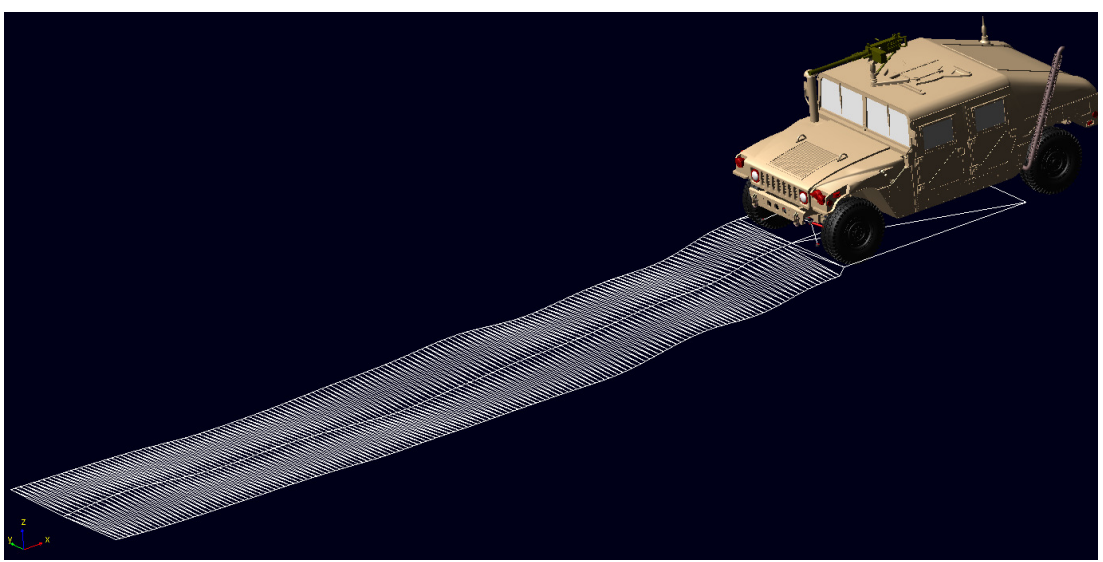

Figure 5. Vehicle on 2 different road surfaces. The two roads are generated using the same training data.

respectively. A set of 200 samples were generated and Figure 5 illustrates two road profiles out of the 200 generated by sampling of the posterior. The road profile was relatively smooth, in the sense that there was no road geometric feature of length comparable to the length of the tire/road contact patch; additionally, the terrain was considered rigid and with a constant

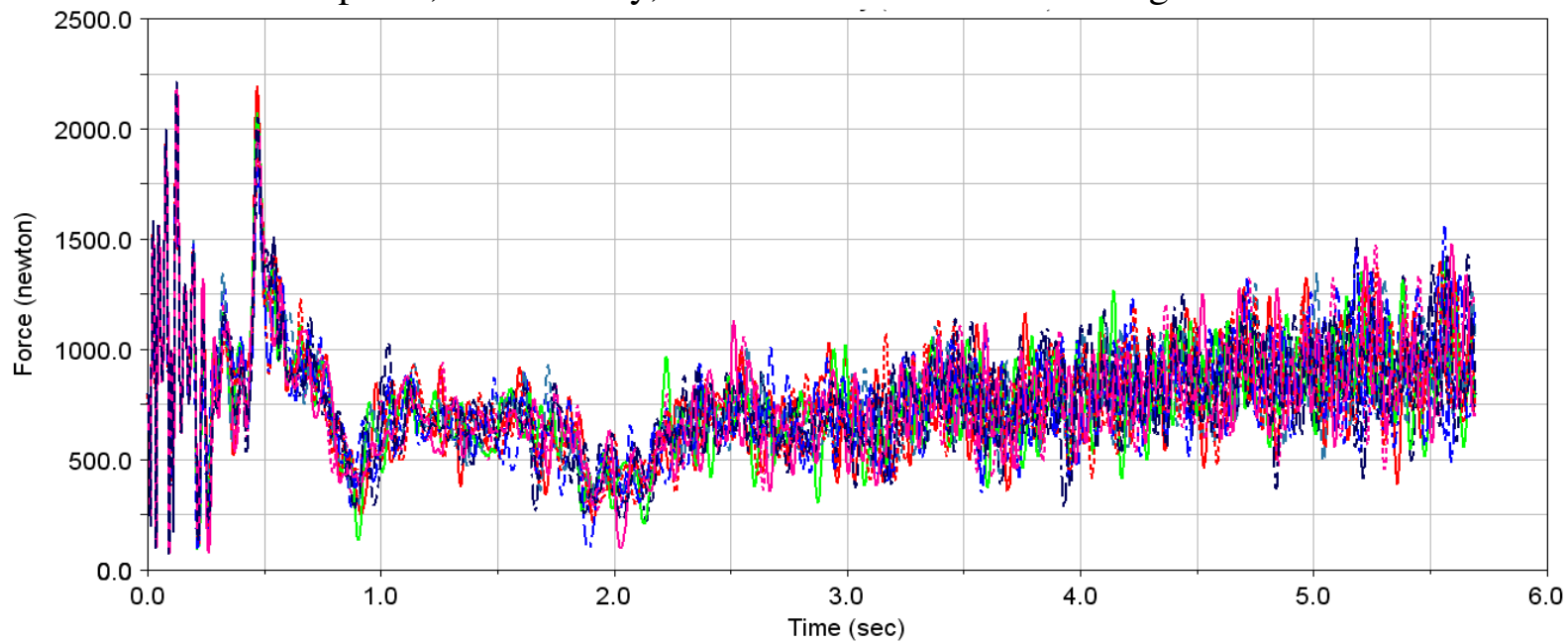

Figure 6. Vehicle response, obtained using ADAMS. Displayed are only a subset of 10 out of the 200 simulations used to generate the statistics of the normal force response. 
friction coefficient.

A batch of 200 ADAMS simulations was subsequently carried out to determine the statistics of the vehicle response. One such response is illustrated in Figure 6, which reports the force response in the bushing connecting the lower control arm (LCA) to the

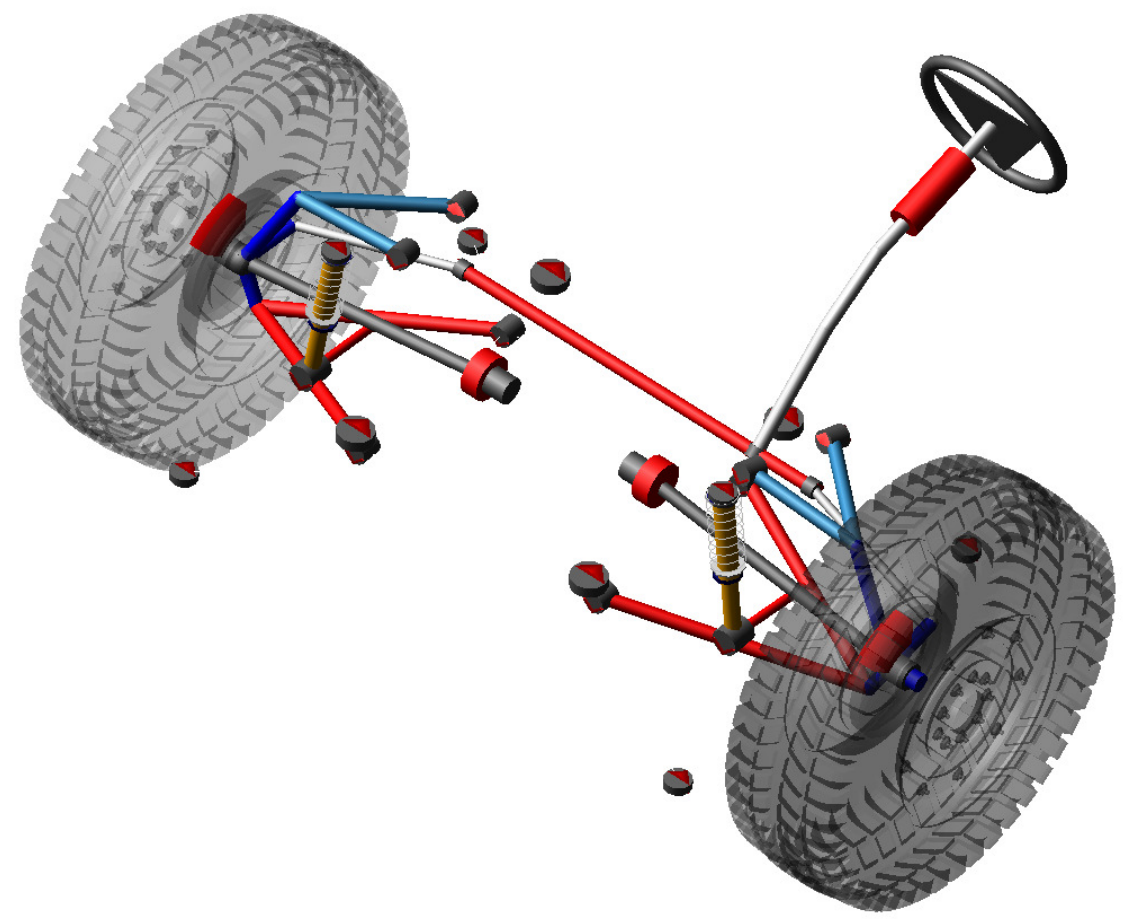

Figure 7: Close up of the suspension system

chassis. There are two such bushings connecting the LCA to the chassis, and there are two more connecting the upper control arm (UCA) to the chassis. There is a grand total

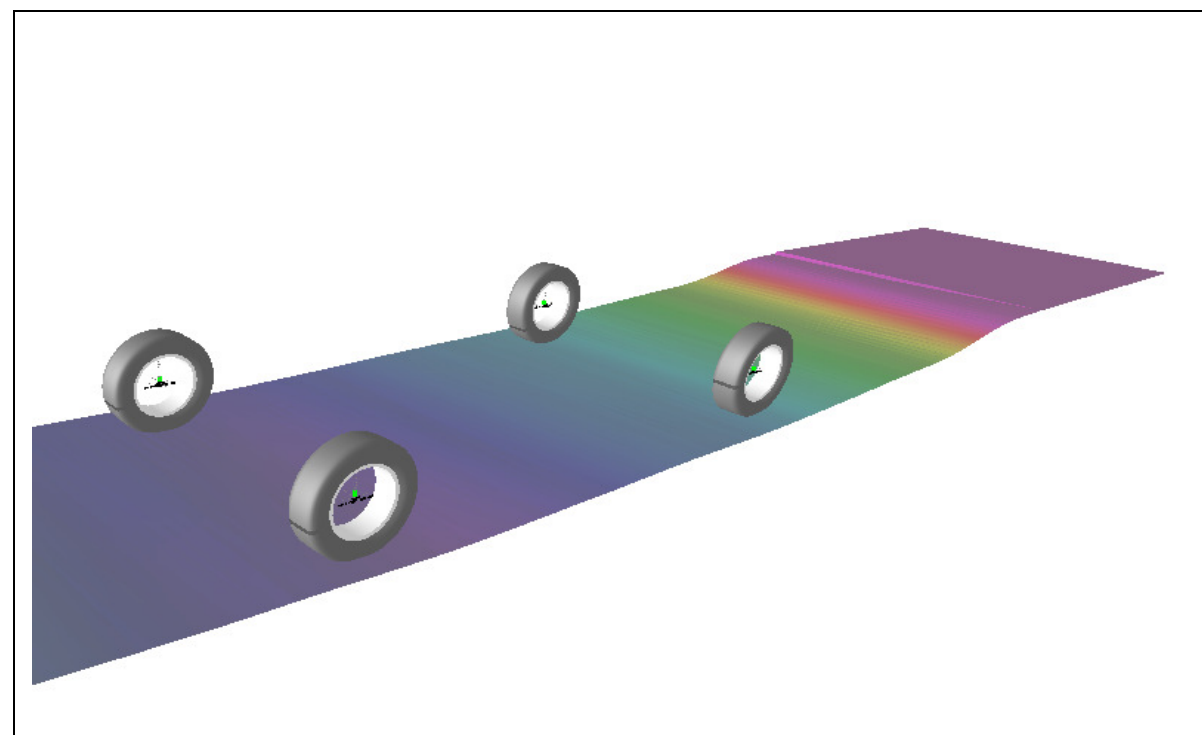

Figure 8: FTire simulation with 4 tires and roads of 16 such bushing elements (eight UCA and eight LCA) in the vehicle model. Average behavior and a 95\% confidence interval are provided for the normal force in the LCA in Figure 10. Note that all results reported in the plots below are in SI units. 


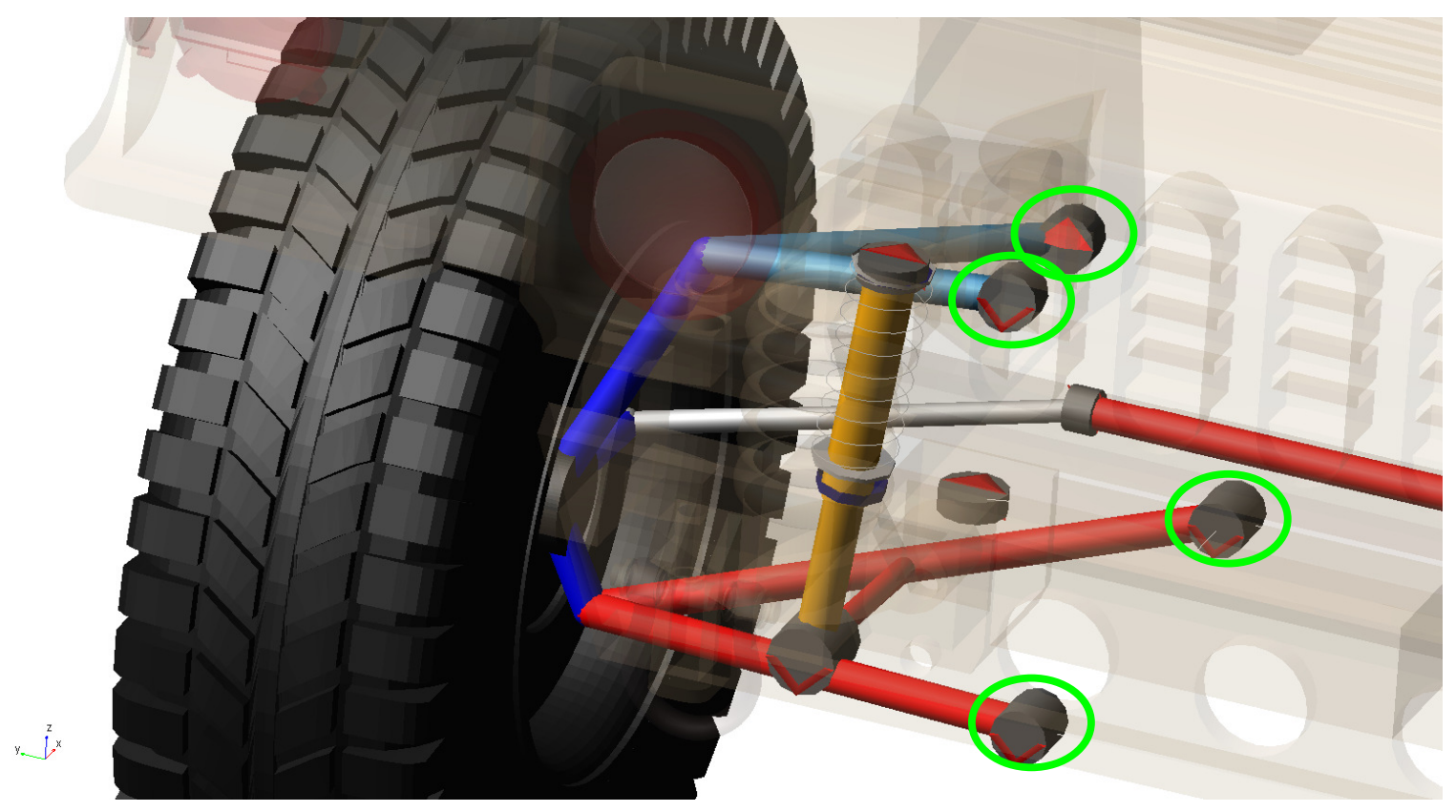

Figure 9: Locations where the force between control arms and the chassis is measured

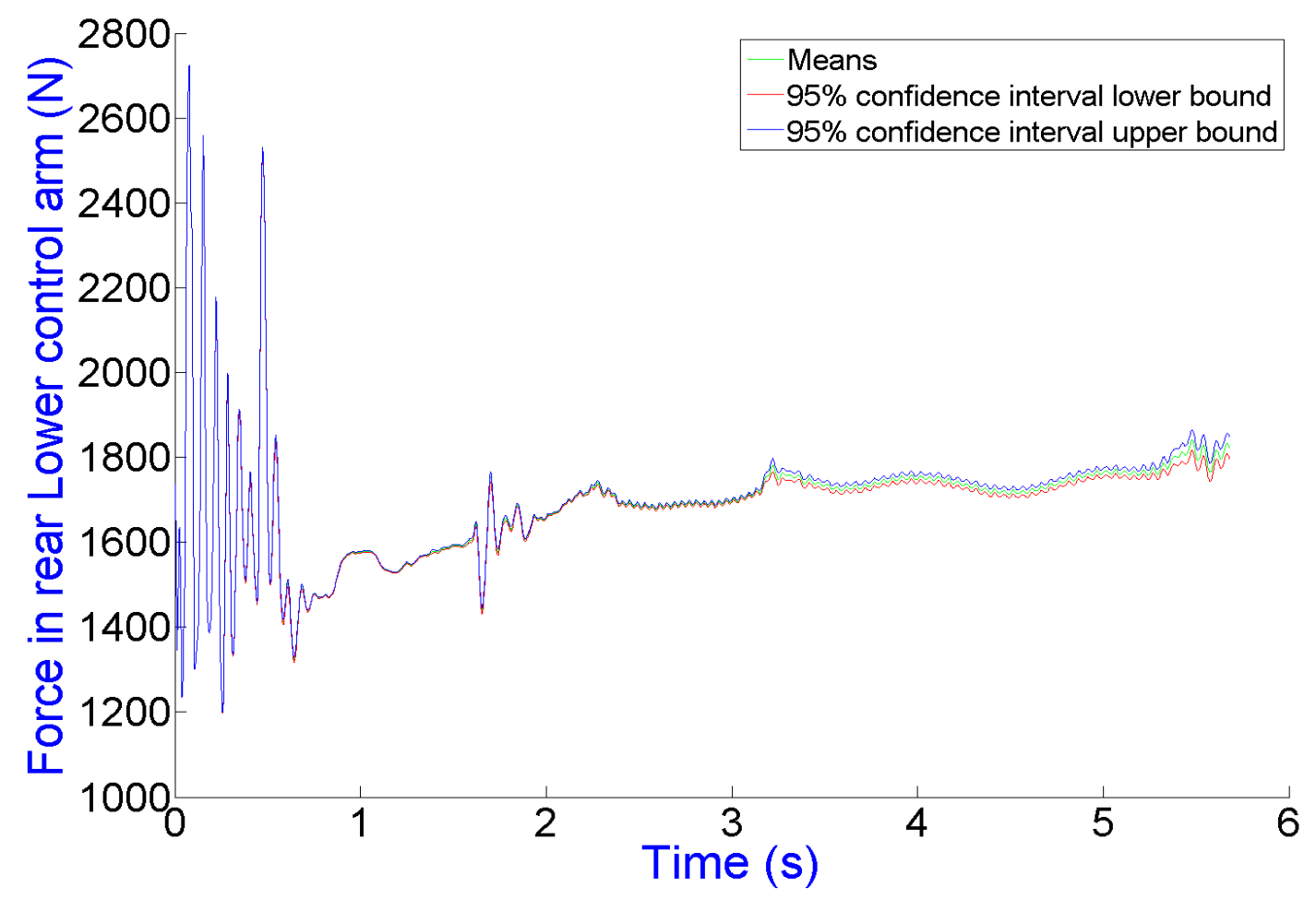

Figure 10. Statistics of vertical load, bushing attached to LCA. 


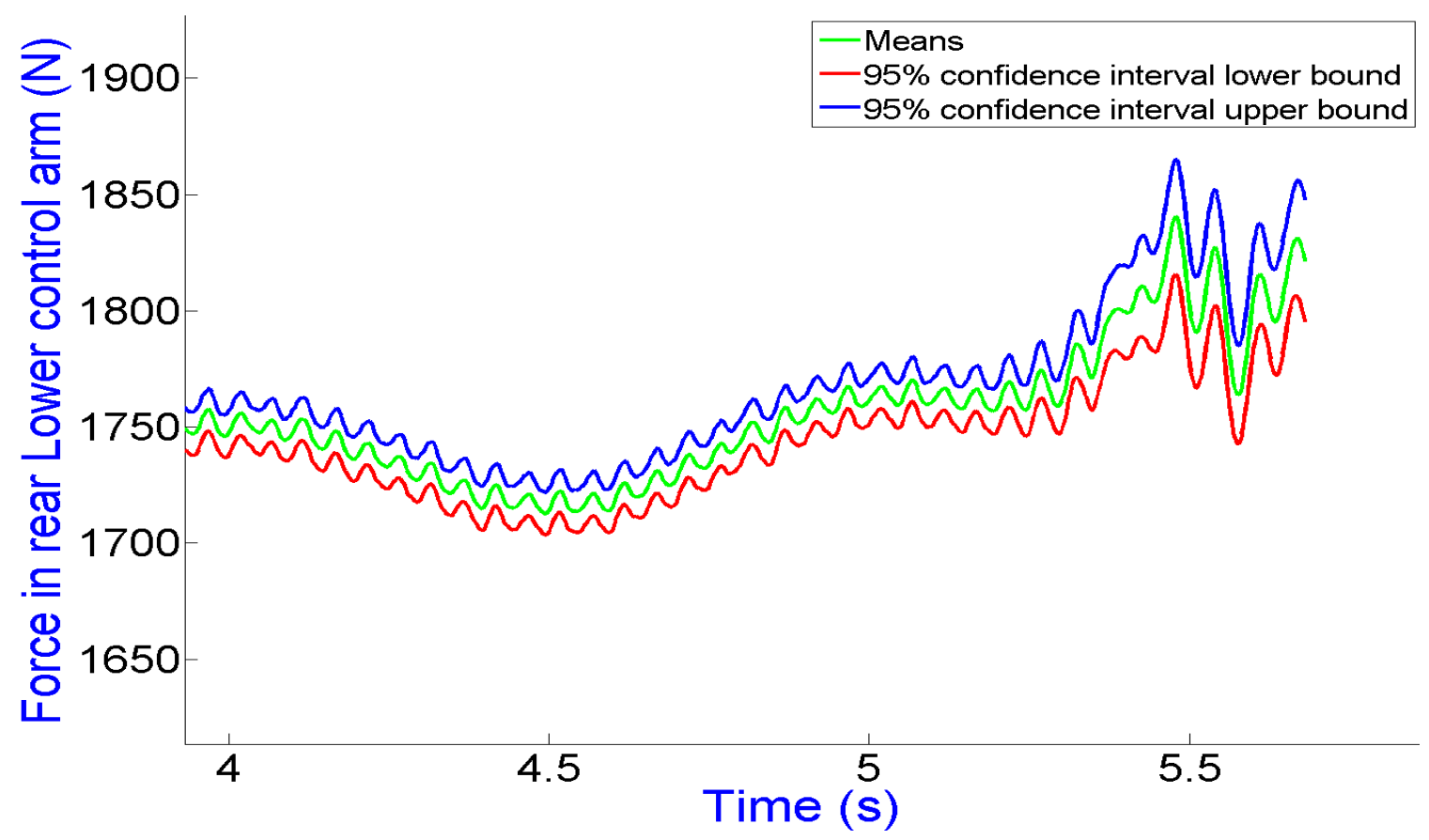

Figure 11. Statistics of vertical load, bushing attached to LCA. Detailed view focused on the last part of run.

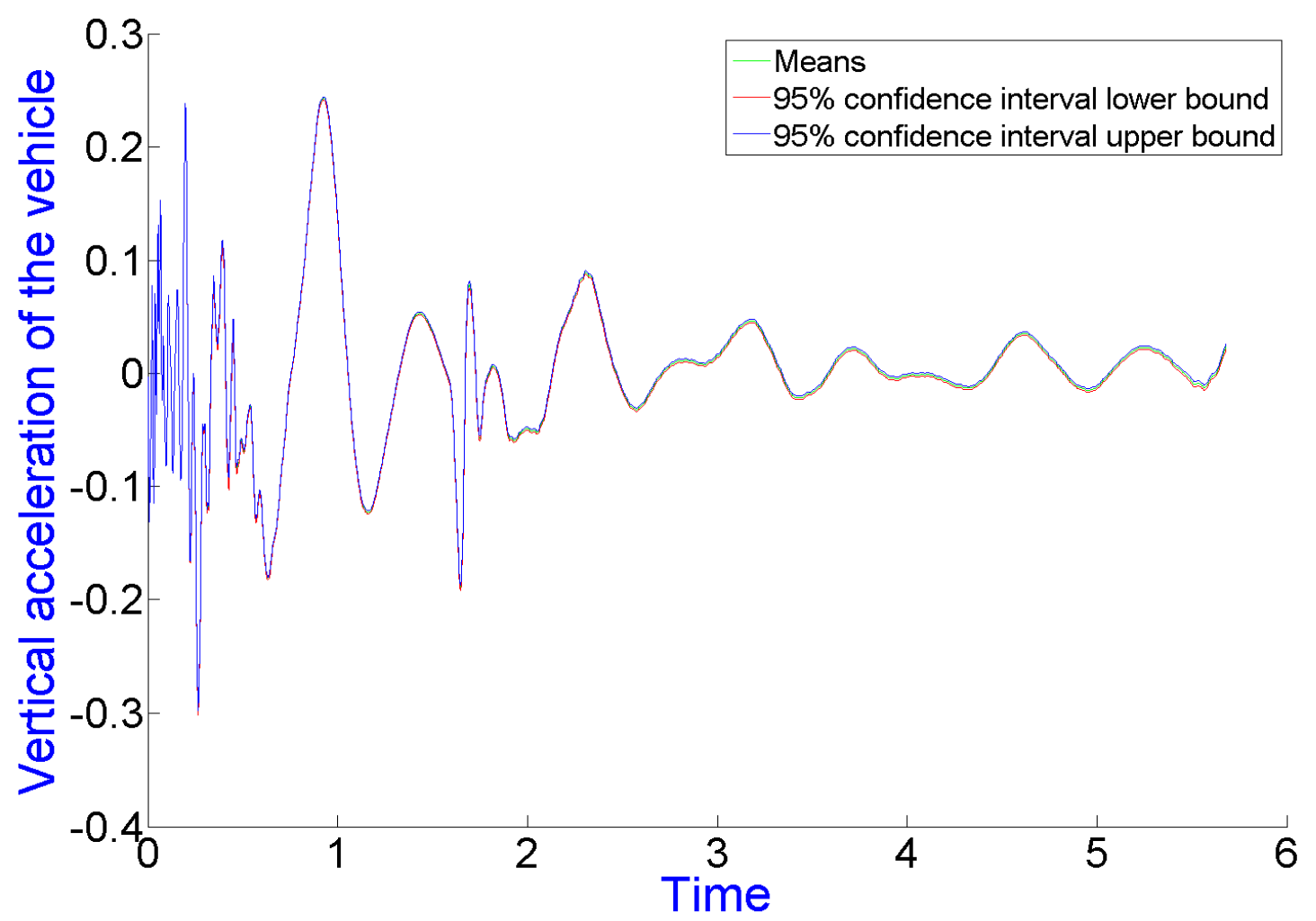

Figure 12. Statistics of vertical acceleration, as measured at the $\mathrm{CG}$ position of the chassis: mean and $95 \%$ confidence interval. 
The results reported in this subsection are obtained setting $\gamma=2$ in Eq. (4). This small change leads to GRF posteriors that lack differentiability. In fact, the SE $(\gamma=1)$ is the only exponential GRF that is continuously differentiable. Figure 13 shows the load

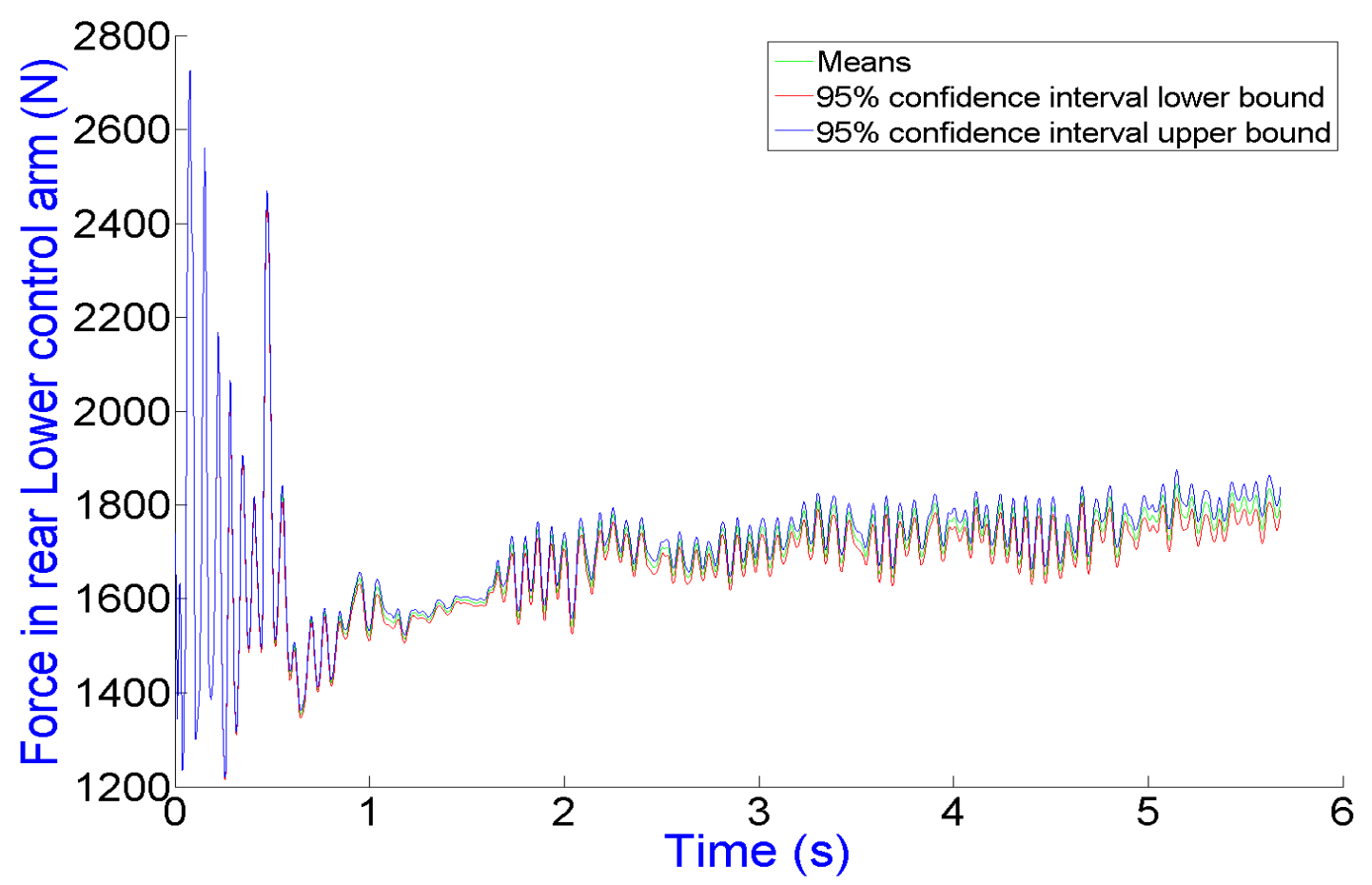

Figure 13. Vertical load statistics, bushing attached to LCA.

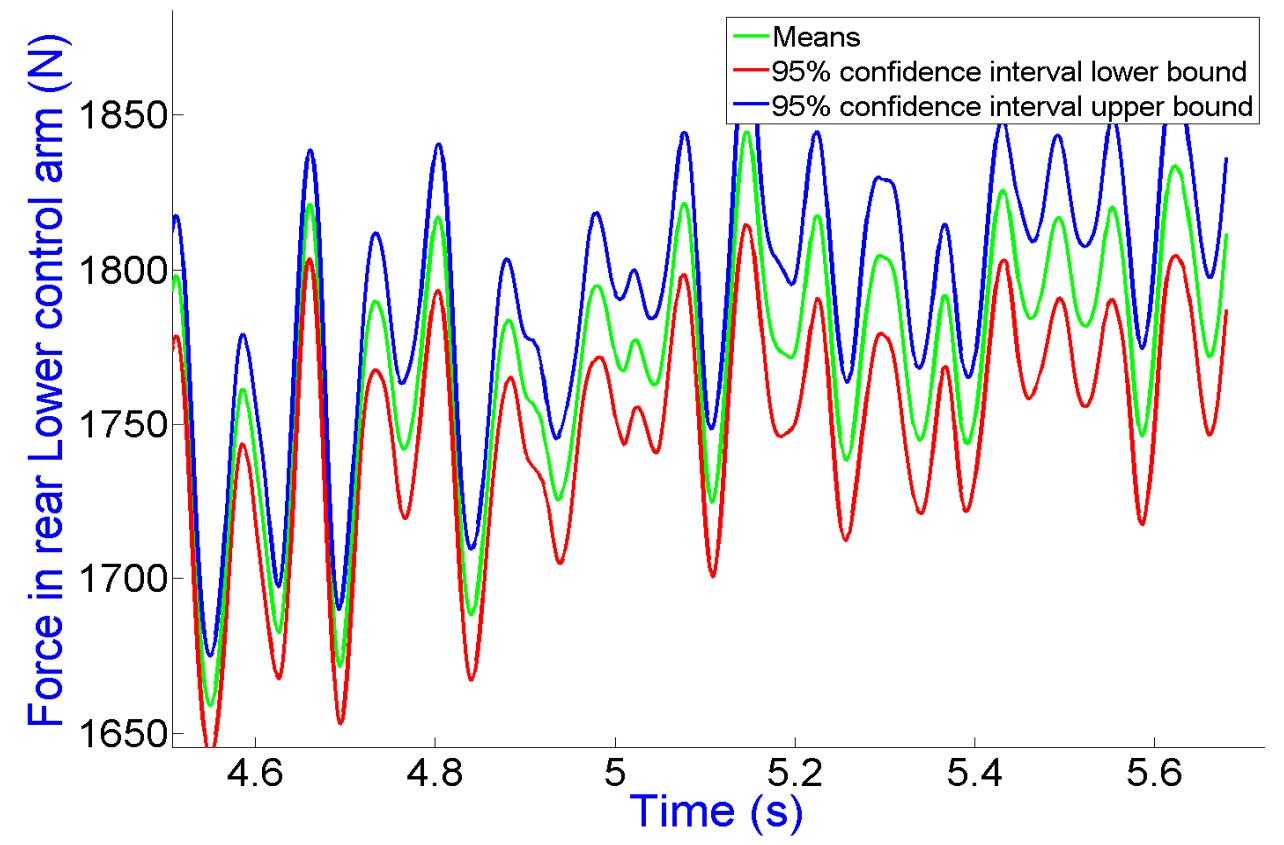

Figure 14. Statistics of vertical load, bushing attached to LCA. Detailed view focused on the last part of run. 
history in the same bushing element as was reported in Figure 10. Figure 14 is a zoom-in to better gauge the $95 \%$ confidence interval for the LCA load. Finally, the vertical acceleration associated with the OU process is reported in Figure 15. Note that for OU

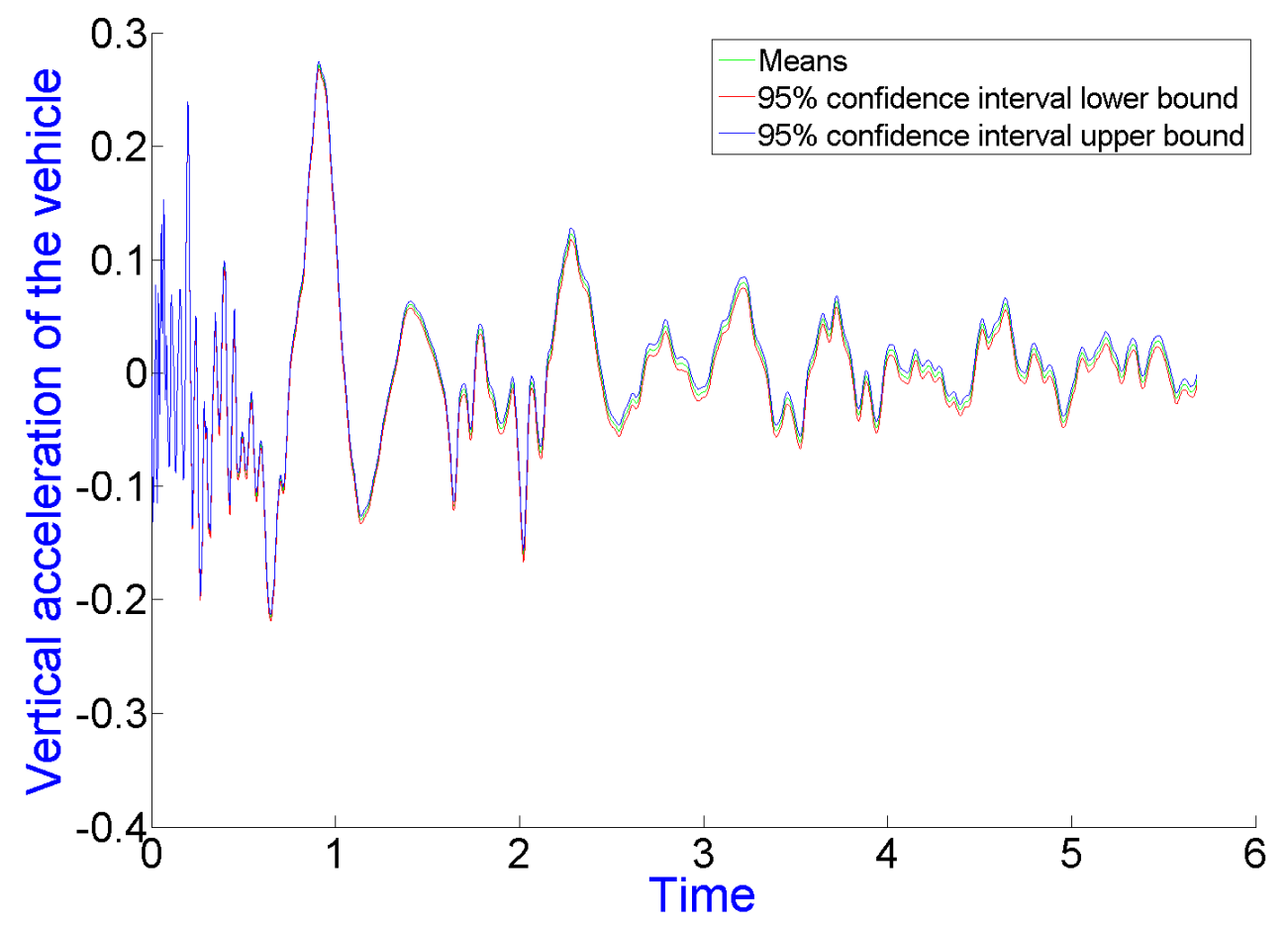

Figure 15. Statistics of vertical acceleration, as measured at the CG position of the chassis: mean and $95 \%$ confidence interval.

the two characteristic lengths are $\theta_{x}=1.0849 \mathrm{e}+003$ and $\theta_{y}=0.1830$.

\section{Discussion of Numerical Results}

The results reported in Figure 11 and Figure 14 suggest that the experimental data is gathered on a dense enough grid because there is relatively small variance in the response of the vehicle. This can be also seen in Figure 11, which illustrates the statistics associated with the last part of the simulation. This is a very good indication that the grid used is sufficiently fine to eliminate a majority of the uncertainty that comes from the road description. In other words, the measured road data is provided at a level of granularity sufficient to pinpoint with good precision the load history for the force acting at a hot point of the LCA.

The results reported in Figure 12 indicate the statistics associated with the vertical acceleration of the center of gravity (CG) of the chassis. In this context, information regarding the vertical acceleration and the jerk measured at a location where the vehicle driver is positioned is valuable as it is used to gauge ride comfort and potential of vibration induced fatigue for long term exposure of a vehicle driver. Although the plot does not report this information at the driver location but rather at the CG location it is 
expected that Figure 12 captures the quantitative trends in the evolution of the vertical acceleration experienced by the driver.

Finally, the comparison of results reported in Figure 10 and Figure 13, or Figure 11 and Figure 14, or Figure 12 and Figure 15 clearly demonstrate the qualitative difference between the SE and OU exponential GRFs. The OU family leads to processes that are nonsmooth, while the SE family leads to road profiles that are continuously differentiable. This difference is reflected in the smoothness of the output: for SE the response is smooth while OU leads to more roughness in the outcome. Nonetheless, there is good agreement between the results obtained with the OU and SE but it remains to further investigate if this roughness associated with OU leads to any significant change in CBM related outcomes. This undertaking falls outside the scope of this study.

\section{Conclusions and future work}

This paper outlines a methodology for predicting the time evolution of complex nonlinear dynamic multi-body system in the presence of uncertain inputs. The focus is on ground vehicle systems, and the associated sources of uncertainty can be multiple: road topology, friction coefficient at the road/tire interface, and aerodynamic force loading. The methodology outlined is general and can be applied to systematically study the impact of sources of uncertainty that were characterized herein by random processes. The scope of the discussion is limited to the case of unknown road profiles at the wheel/road interface of a military vehicle. The same approach can be used in conjunction with vehicle design parameters by substituting the GRF machinery with that of sampling from a random variable distribution. The latter scenario is simpler and not discussed here. It would closely follow the methodology outlined in Figure 1 in the sense that one could deal with parametric or nonparametric models, and then invoke the same MLE based learning stage for defining the posterior distribution. The latter is subsequently sampled in a Monte-Carlo analysis to gauge the impact of uncertainty on the dynamics of the model.

The results reported herein suggest that the choice of correlation function is important. Further work is needed to better understand the sensitivity of the system response to the correlation function. In this context, two directions of future work could prove insightful. First, it would be useful to compare other GRF correlation functions, both stationary and non-stationary, to further understand the importance of this choice. Second, it would be useful to lift the requirement that the stochastic approach be molded into the GRF idea. Nonparametric models should be considered and the benefits that come from improved flexibility should be weighted against the more involved analytical, method implementation, and computational burden associated with such an approach.

Even before considering nonparametric techniques the computational burden associated with the methodology proposed herein can be already significant. This is particularly the case when the amount of learning data is vast and/or the posterior is sampled on a very fine grid. This issue can be addressed by either allocating more 
computational power to solve the problem, or by considering new sampling techniques. The increased computational power aspect is currently under investigation (Datar 2008). Recent inroads into better sampling techniques have been recently reported by Anitescu, Schmitt, et. al (2008), where a substantial reduction in sampling effort is demonstrated by the use of two new approaches. The first makes a periodicity assumption that enables a Fast Fourier Transform (FFT) technique to be involved, while the second uses compact kernel covariance functions to sample small sliding windows that are continuously moved over the region of interest.

\section{Acknowledgements}

D. Negrut would like to thank BAE Systems for providing partial financial support that allowed his participation in this research effort. Dr. M. Anitescu, K. Schmitt, and J. Madsen are acknowledged for their valuable suggestions. 


\section{References}

Adler, R. J., 1990: An Introduction to Continuity, Extrema, and Related Topics for General Gaussian Processes. Institute of Mathematical Statistics.

Anitescu, M., K. Schmitt, and D. Negrut, 2008: Efficient sampling of dynamical systems with spatial uncertainty. International Journal for Numerical Methods in Engineering, submitted.

Cramér, H., and M. R. Leadbetter, 1967: Stationary and related stochastic processes. Wiley

Datar, M., 2008: Uncertainty Quantification in Ground Vehicle Simulation, M.S. Thesis, Mechanical Engineering, University of Wisconsin-Madison.

Gipser, M., 2005: FTire: a physically based application-oriented tyre model for use with detailed MBS and finite-element suspension models. Vehicle Systems Dynamics, 43, $76-91$.

Hairer, E., and G. Wanner, 1996: Solving Ordinary Differential Equations II: Stiff and Differential-Algebraic Problems. Second Revised ed. Vol. 14, Springer.

Haug, E. J., 1989: Computer-Aided Kinematics and Dynamics of Mechanical Systems. Volume I:Basic Methods. Allyn and Bacon.

Matérn, B., 1960: Spatial Variation (Lecture NotesStatist. 36). Springer, Berlin.

Neal, R., 1996: Bayesian Learning for Neural Networks. Springer.

Rasmussen, C. E., and C. K. I. Williams, 2006: Gaussian processes for machine learning. Springer.

Santner, T. J., B. J. Williams, and W. Notz, 2003: The Design and Analysis of Computer Experiments. Springer.

Schmitt, K., J. Madsen, M. Anitescu, and D. Negrut, 2008: A Gaussian Process Based Approach for Handling Uncertainty in Vehicle Dynamics Simulations - IMECE200866664. 2008 ASME International Mechanical Engineering Congress and Exposition, Boston, MA, ASME.

Stein, M., 1999: Interpolation of Spatial Data: Some Theory for Kriging. Springer.

Uhlenbeck, G., and L. Ornstein, 1930: On the Theory of the Brownian Motion. Physical Review, 36, 823-841.

Wasserman, L., 2006: All of Nonparametric Statistics. Springer.

Xiu, D., and G. E. Karniadakis, 2002: The Wiener-Askey Polynomial Chaos for Stochastic Differential Equations. SIAM Journal on Scientific Computing, 24, 619644. 